\title{
Archéopages
}

Archéopages

Archéologie et société

$36 \mid 01 / 2013$

Exotismes

\section{Un regard porté sur l'ailleurs. Céramiques à Lyon entre Moyen Âge et temps modernes}

\section{Alban Horry}

\section{(2) OpenEdition}

1 Journals

Édition électronique

URL : https://journals.openedition.org/archeopages/213

DOI : 10.4000/archeopages. 213

ISSN : 2269-9872

Éditeur

INRAP - Institut national de recherches archéologiques préventives

Édition imprimée

Date de publication : 1 novembre 2013

Pagination : 36-47

ISSN : 1622-8545

\section{Référence électronique}

Alban Horry, « Un regard porté sur l'ailleurs. Céramiques à Lyon entre Moyen Âge et temps

modernes ", Archéopages [En ligne], 36 | 01/2013, mis en ligne le 01 janvier 2015, consulté le 21 janvier 2022. URL : http://journals.openedition.org/archeopages/213 ; DOI : https://doi.org/10.4000/ archeopages. 213 


\title{
Un regard porté sur l'ailleurs Céramiques à Lyon entre Moyen Age et temps modernes
}

\author{
Alban Horry Inrap
}

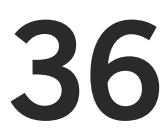

1. Fouille, dir. G. Ayala, Inrap, 2002-2004.

2. Identification MarieOdile Rousset (Gremmo) 3. Identification Luis de La Revilla Negro (Museo Nacional de Arte Hispanomusulmán, Grenade-Espagne). 4. Fouille, dir. C. Bellon, Afan, 1996.
Aborder le thème de l'exotisme et des céramiques est un exercice difficile. Les données concernant ce goût pour l'étranger sont abondantes, et dans des domaines qui vont bien au-delà de celui de l'archéologie. On se bornera ici à évoquer ce que les sources archéologiques lyonnaises, constituées par des milliers de tessons de céramique, apportent comme supposées preuves matérielles de cet engouement. Il est délicat, dans certains cas, de déterminer si ces découvertes témoignent d'un intérêt et d'une volonté réelle d'acquérir des objets exotiques, ou si elles sont l'expression de rapports commerciaux depuis des contrées éloignées. Force est de constater que le Moyen Âge et le début de la période moderne, à Lyon en tout cas, n'attestent que très timidement d'éventuelles affinités pour des céramiques issues de pays lointains, et pas seulement en Orient. Il est difficile d'imaginer la puissance de l'exotisme véhiculée par les céramiques et la fascination qu'elles pouvaient alors susciter. C'est assurément à partir de la fin du $\mathrm{XVII}^{\mathrm{e}}$ siècle et surtout au XVIII ${ }^{\mathrm{e}}$ siècle que les produits en terre cuite semblent combler un goût croissant pour l'ailleurs, en lien avec un phénomène culturel plus vaste.

\section{Peut-on parler d'exotisme au Moyen Âge?}

À Lyon, le Moyen Âge fait un peu figure de parent pauvre quand il s'agit d'analyser l'arrivée de céramiques dites exotiques. En effet, depuis les environs de l'an mil, les vaisselles domestiques locales sont caractérisées par une certaine austérité que leur confère cet aspect uniformément gris des céramiques communes d'origine régionale. Quelques rares découvertes attestent la présence d'objets venus de contrées lointaines à partir des $\mathrm{XIII}^{\mathrm{e}}-\mathrm{XIV}^{\mathrm{e}}$ siècles. C'est le cas des vases issus du
Proche-Orient : un albarello sur le site de LyonTramassac (Thiriot, 1991) et les fragments d'un vase découvert à Lyon, parc Saint-Georges ${ }^{1}$ (Ayala, 2013, p. 179) sans doute originaire de Syrie ou du Liban ${ }^{2}$. Difficile néanmoins de déterminer si ces récipients témoignent d'une volonté d'acquérir des objets précieux et et qui sortent du commun ; ils sont plus probablement les emballages d'épices ou denrées rares (Thiriot, 1995, p. 28-29) destinées à quelque apothicaire ou marchand lyonnais. Vers la fin du Moyen Âge, on note çà et là quelques éléments rares, issus des bords de la Méditerranée. Quoi de plus exotique, par exemple, que cette grande jarre arabo-andalouse $\mathbf{3}^{\mathbf{3}}$ au décor à la cuerda seca [ill. 1] trouvée à Lyon, dans le quartier de Vaise ${ }^{\mathbf{4}}$, dans un dépotoir du xiv ${ }^{\mathrm{e}}$ siècle (Horry, 2001, p. 138141) ? Les écuelles en faïence au décor de lustre de cuivre des ateliers valenciens de Manises et Paterna sont également bien discrètes (Horry, 2002). Avec seulement quelques individus découverts, on est en effet ici bien loin de l'abondance que l'on observe dans les villes provençales ou languedociennes. Tout au plus, ces petits récipients constituent les premières faïences disponibles sur le marché, et il n'est pas impossible qu'elles aient été des vaisselles acquises pour le luxe qu'elles représentent. Leurs reflets cuivrés ont pu susciter un intérêt de la part de consommateurs encore peu habitués, semble-t-il, à ce genre de céramique. À Lyon, l'uniformité des vaisselles du Moyen Âge est progressivement atténuée à partir du XIII ${ }^{\mathrm{e}}$ siècle par l'apparition de nouveaux récipients en céramique à pâte rouge recouverts de glaçure plombifère. Majoritairement destinés à la consommation des boissons, ils sont sans doute de fabrication locale ou régionale. Ils sont ornés à la façon des objets des pays du Nord. Faut-il y voir une imitation qui évoque un goût certain pour ces 
1. Un petit témoignage arabo-andalou ; fragment d'une grande jarre ou jarrita en céramique andalouse décorée " a la cuerda seca ॥

retrouvé piégé dans les

sables d'une rivière tout près de Lyon (hauteur $3 \mathrm{~cm}$ ).

2. Pichet tonnelet en

céramique rouge glaçurée

du XIve siècle découvert dans

un dépotoir lyonnais; objet

ostentatoire destiné à orner

quelque dressoir (hauteur

$22 \mathrm{~cm})$.
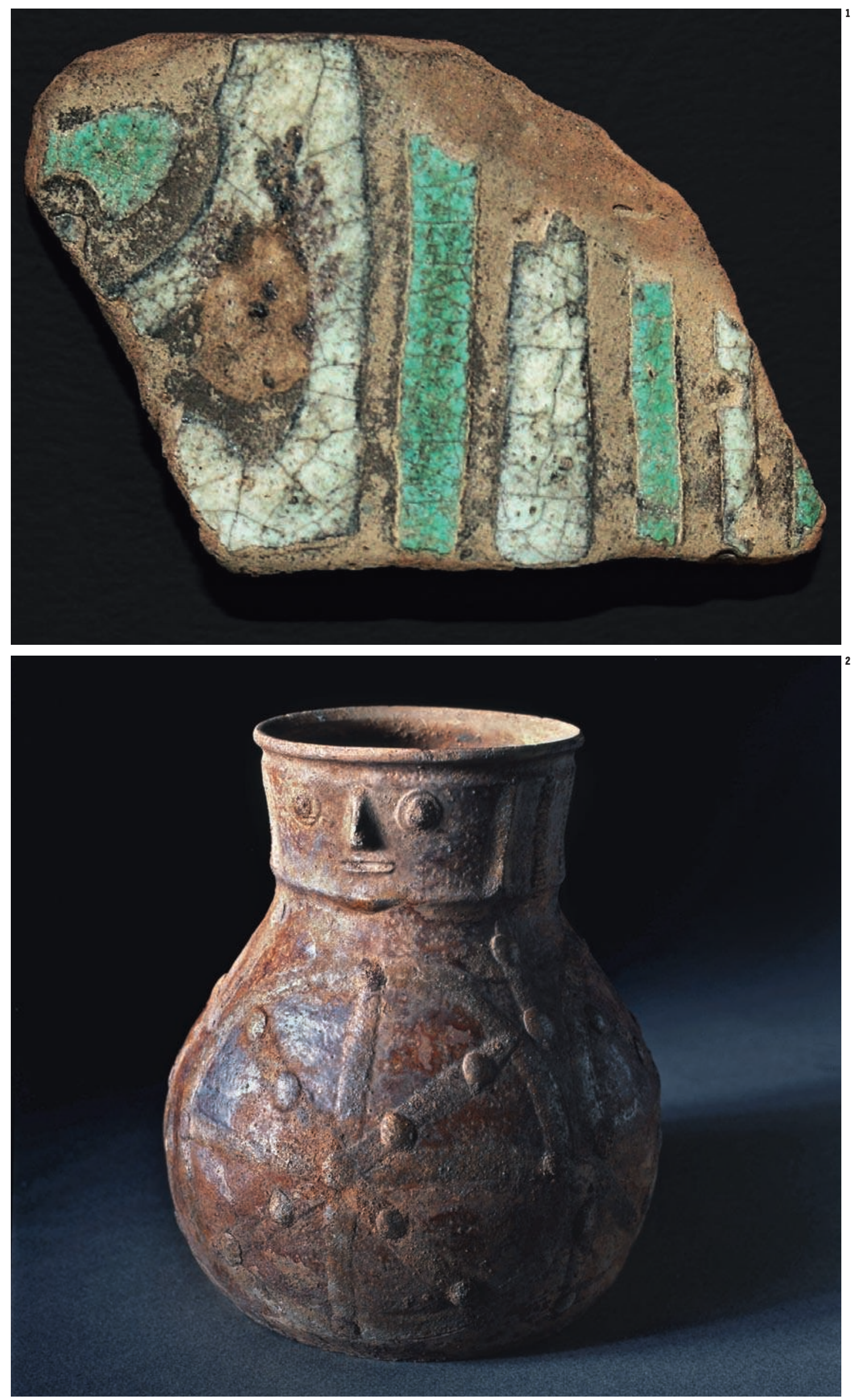


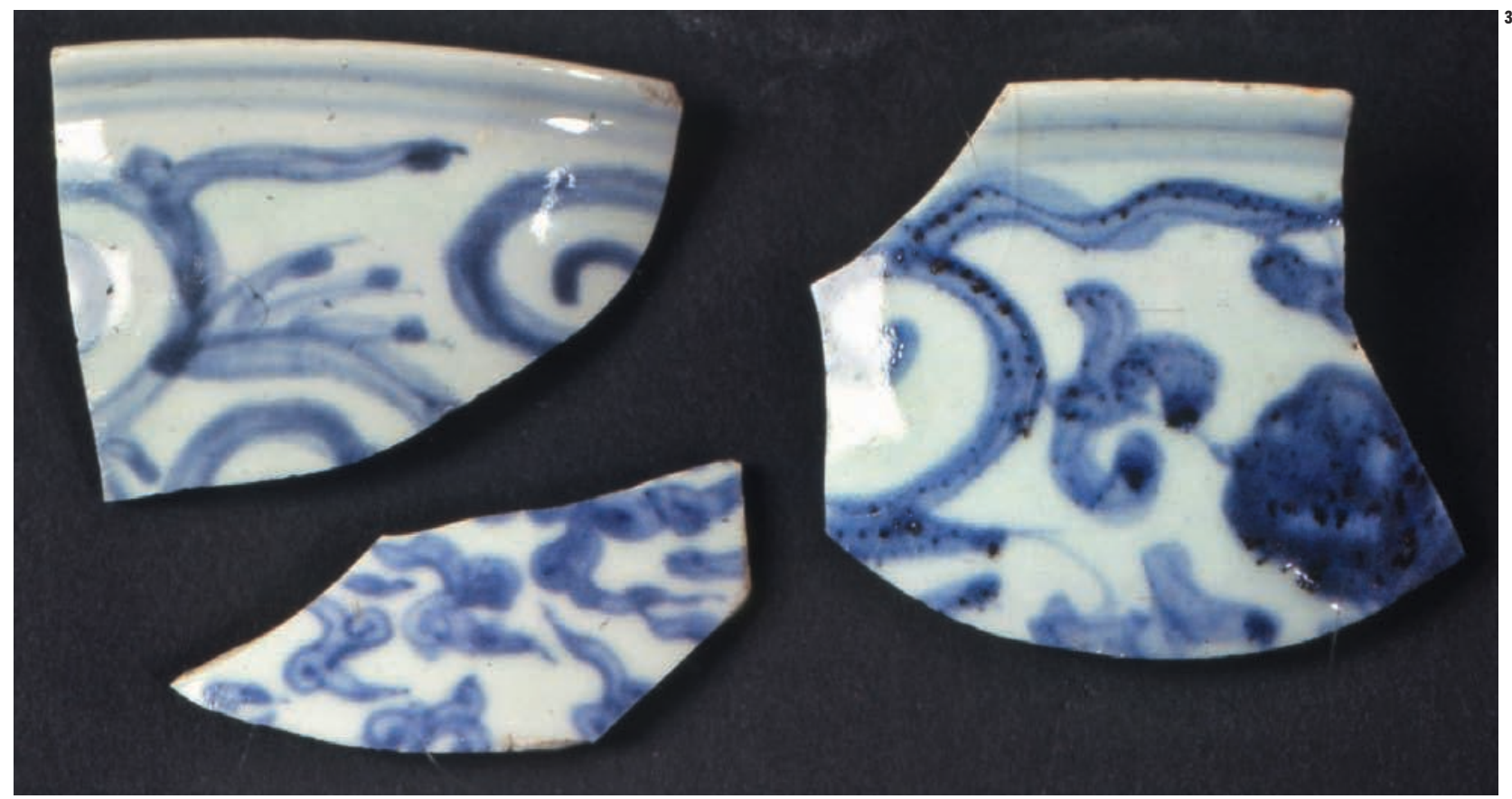

3 et 4. Les porcelaines Ming et grès céladon atteignent

la ville de Lyon dès le xive siècle

découvertes pour cette

période dans la région

(hauteur maximum $5 \mathrm{~cm}$ pour

le $n^{0} 3$; hauteur $8,5 \mathrm{~cm}$ pour

le $n^{0} 4$ ).

5. Retrouvé dans les tourbes

des berges de la Saône,

ce fragment de plat en

céramique ottomane d'Iznik

est le premier témoignage

archéologique de l'importation

de cette production

prestigieuse à Lyon (hauteur

$6 \mathrm{~cm}$, largeur $10 \mathrm{~cm}$ ).

6. Ce petit fragment de chope

en céramique ottomane

constitue à lui seul un

échantillon significatif du

registre décoratif des potiers

d'Iznik du xvI 'eiècle avec une

tulipe et un œillet (hauteur

$6 \mathrm{~cm})$.

7. Le dépotoir de la seconde moitié du xvil' siècle sur les

berges de la Saône (Lyon-

Saint-Georges) a livré un

petit lot de porcelaines

chinoises et japonaises.

Certains fragments (tasses,

sous-tasses) appartiennent

assurément à des objets

destinés à la consommation

des boissons exotiques

thé, café, chocolat pénètrent

alors les habitudes

de consommation depuis

près d'un siècle.
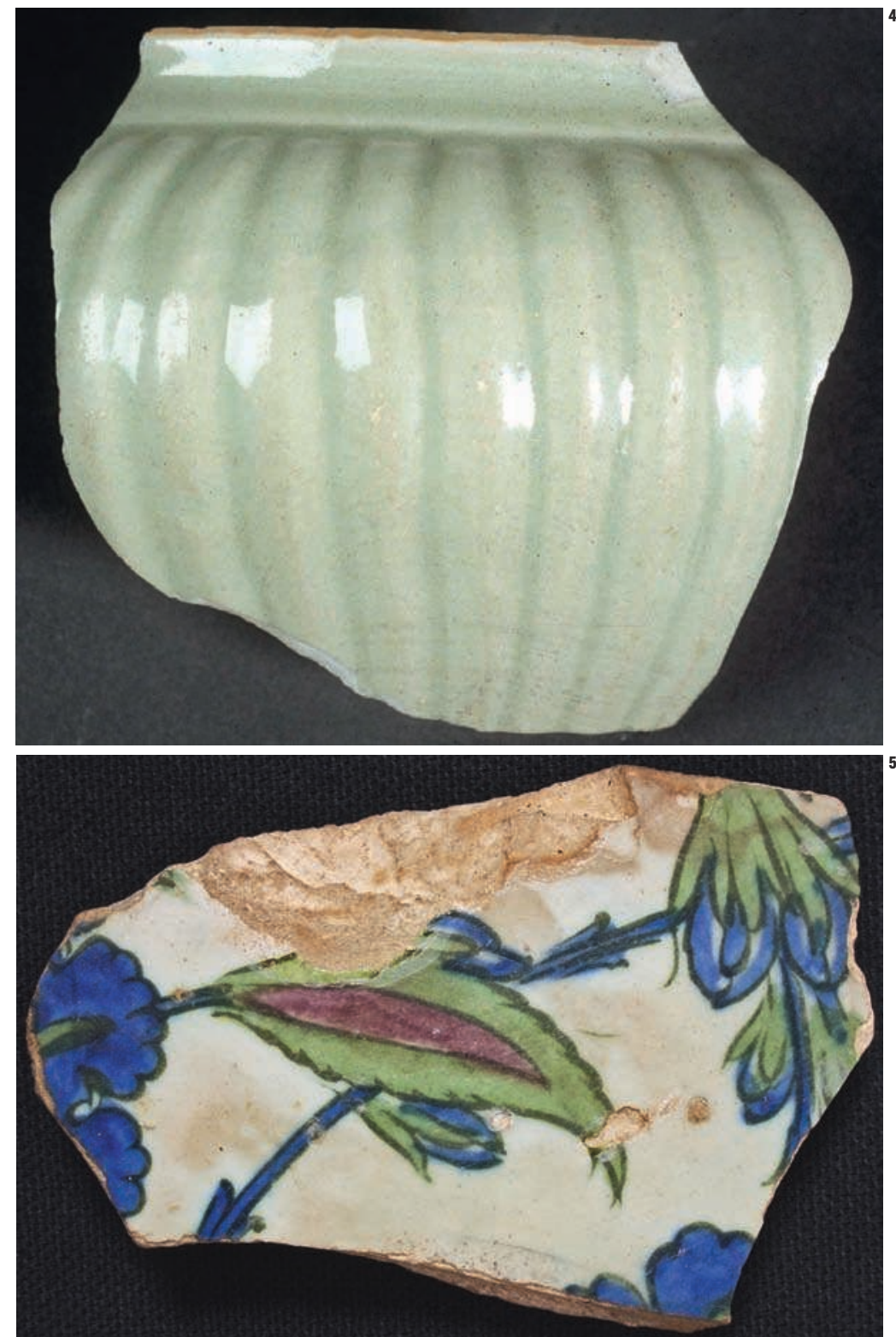
objets dits « très décorés » (Horry, 2009), peut-être exotiques au regard des Lyonnais? Ce pourrait être le cas des grands pichets tonnelets [ill. 2] au profil insolite dont on trouve de nombreux parallèles dans les Flandres ou en Grande-Bretagne, et que les sites lyonnais livrent régulièrement. La situation géographique de Lyon au cœur de diverses influences culturelles est d'ailleurs assez significative lorsqu'il s'agit d'étudier les objets du quotidien en terre cuite à la période médiévale (Horry, 2012a).

\section{$\mathrm{XVI}^{\mathrm{e}}$ siècle : découverte de nouveaux styles}

$\mathrm{Au} \mathrm{xvI}^{\mathrm{e}}$ siècle, Lyon est souvent qualifiée de « capitale des épices ». Ces denrées, importées d'Orient via l'Italie, arrivent par le sud de la France ou par les Alpes. On peut supposer que les petits bols blancs et bleus en porcelaine de Chine d'époque Ming et les grès céladon [ill. 3 et 4], découverts dans le vaste dépotoir collectif du site de la place des Terreaux (Lyon $1^{\mathrm{er}}$ ), ont suivi les mêmes routes, ou bien celle si prestigieuse de la soie. Leur rareté en fait surtout des pièces de grand luxe, destinées à une population aisée, et le nombre si peu élevé de découvertes ne laisse pas supposer un phénomène de commerce de haute densité. Il semble que l'on puisse parler, comme au Moyen Âge, de cadeaux, d'objets ramenés par leurs propriétaires, ou encore de petits récipients faisant partie d'une cargaison de produits importés d'Extrême-Orient. Les céramiques des XVI ${ }^{\mathrm{e}}-\mathrm{XVII}{ }^{\mathrm{e}}$ siècles originaires des contrées orientales plus proches sont également rarissimes. À Lyon, on ne connaît à ce jour qu'une seule découverte, à SaintGeorges, d'un fragment de plat des ateliers ottomans de la région d'Iznik, en Turquie [ill. 5]. On citera également, un petit morceau de chope [ill. 6], exhumé du fossé d'un château ${ }^{5}$ du pays de Gex (Ain), près de Genève. Ces tessons constituent de rares attestations archéologiques de ces céramiques de grand luxe en France, avec les cas marseillais (Amouric et al., 1999, p. 112-115), qui étaient l'apanage des milieux aristocratiques.

Peut-on attribuer les changements qui s'opèrent vers le milieu du Xvi ${ }^{\mathrm{e}}$ siècle à Lyon, dans le domaine des céramiques du quotidien, à un goût pour des objets différents? Les styles rappellent de près ceux du sud de la France et parfois de l'Italie. La présence de fortes communautés étrangères dès la fin du Moyen Âge, en particulier italiennes, a dû probablement véhiculer des innovations et des objets jusqu'alors inconnus. D'une part, les artisans lyonnais de la terre cuite ont sans doute répondu à des demandes émanant de ces communautés diverses. D'autre part, l'installation de potiers originaires de Toscane dès les années $1510 \mathrm{a}$ pu influencer la production de vaisselles aux riches décors et aux techniques novatrices (Amouric et $a l ., 2009)$. En effet, les études menées au gré des découvertes archéologiques confirment l'influence notable des majoliques italo-lyonnaises sur les autres productions, qui leur donnent un « air
d'Italie » (Horry, 2012b, p. 40-50). Enfin, l'arrivée régulière, grâce à l'essor commercial de Lyon, de produits issus de contrées plus au moins éloignées aura sans doute également sa part d'influence sur le goût des consommateurs locaux. Il semble ici que c'est plutôt l'offre qui suscite un intérêt croissant pour de nouveaux produits, peut-être à l'origine de courants de modes. Il reste difficile néanmoins de déterminer si l'émergence de toute une catégorie de produits relève du simple attrait de la nouveauté ou si elle est liée à un goût pour « l'exotisme » de vaisselles venues d'ailleurs. Cette découverte de nouveaux styles est aussi parfois transmise par des vaisselles importées, et on citera par exemple les majoliques ligures, mises au jour sur plusieurs sites lyonnais. Ces produits à l'émail bleu et aux décors fins caractéristiques sont ornés de motifs mêlant les influences des porcelaines chinoises et des céramiques ottomanes d'Iznik, relevant d'un Orient que l'on peut qualifier de fabuleux.

\section{$\mathrm{XVII}^{\mathrm{e}}$ et $\mathrm{XVIII}^{\mathrm{e}}$ siècles : influence de l'Orient}

C'est à partir de la fin du XVII ${ }^{\mathrm{e}}$ siècle et au XVIII ${ }^{\mathrm{e}}$ siècle que les porcelaines de Chine et du Japon sont le plus diffusées en France grâce au développement du grand commerce maritime. Destinées à des acheteurs fortunés, leur apparition est aussi intimement liée à celle des boissons « exotiques », café, thé et chocolat. Les porcelaines sont encore très rares sur les sites lyonnais. Les quelques individus découverts à Saint-Georges (Lyon $5^{\mathrm{e}}$ ) sont sans doute pour la plupart originaires de Chine, avec des assiettes aux décors en camaïeu bleu [ill. 7]. On trouve également des exemplaires aux motifs vert et rose, des produits distribués par le biais de la Compagnie des Indes, et le Japon se signale par quelques petites coupes originaires d'Imari. Ces porcelaines vont avoir une influence notable sur les productions européennes à la fois dans les motifs, mais également dans les formes. L'importation, dans de très grandes quantités, de ces produits d'Extrême-Orient, par la Compagnie des Indes, suscite copies et interprétations dès la fin du XVII ${ }^{\mathrm{e}}$ siècle. Mais Lyon ne vit pas l'implantation de fabriques de porcelaine. Les découvertes dans les dépotoirs lyonnais de la seconde moitié du XVII ${ }^{\mathrm{e}}$ siècle et surtout du XVIII ${ }^{\mathrm{e}}$ siècle permettent d'aborder l'influence des produits de l'Orient, surtout perceptible dans le domaine de la faïence, qui, grâce à l'essor des manufactures à Lyon et dans d'autres régions de France, va permettre la diffusion, et sur de longues distances, de produits d'une extrême variété. Ces derniers vont être des vecteurs de circulation des modes et des styles décoratifs dans lesquels l'attrait pour l'exotisme et le goût pour l'ailleurs vont pouvoir amplement s'épanouir.

On peut dire que les exemples livrés par l'archéologie sont des objets à la mode qui développent un imaginaire tourné vers les contrées lointaines. L'attrait de la nouveauté et de l'exotisme passe par contenants et contenus (les boissons), et
5. Fouille, dir. S. Motte, Afan, 1995 . 


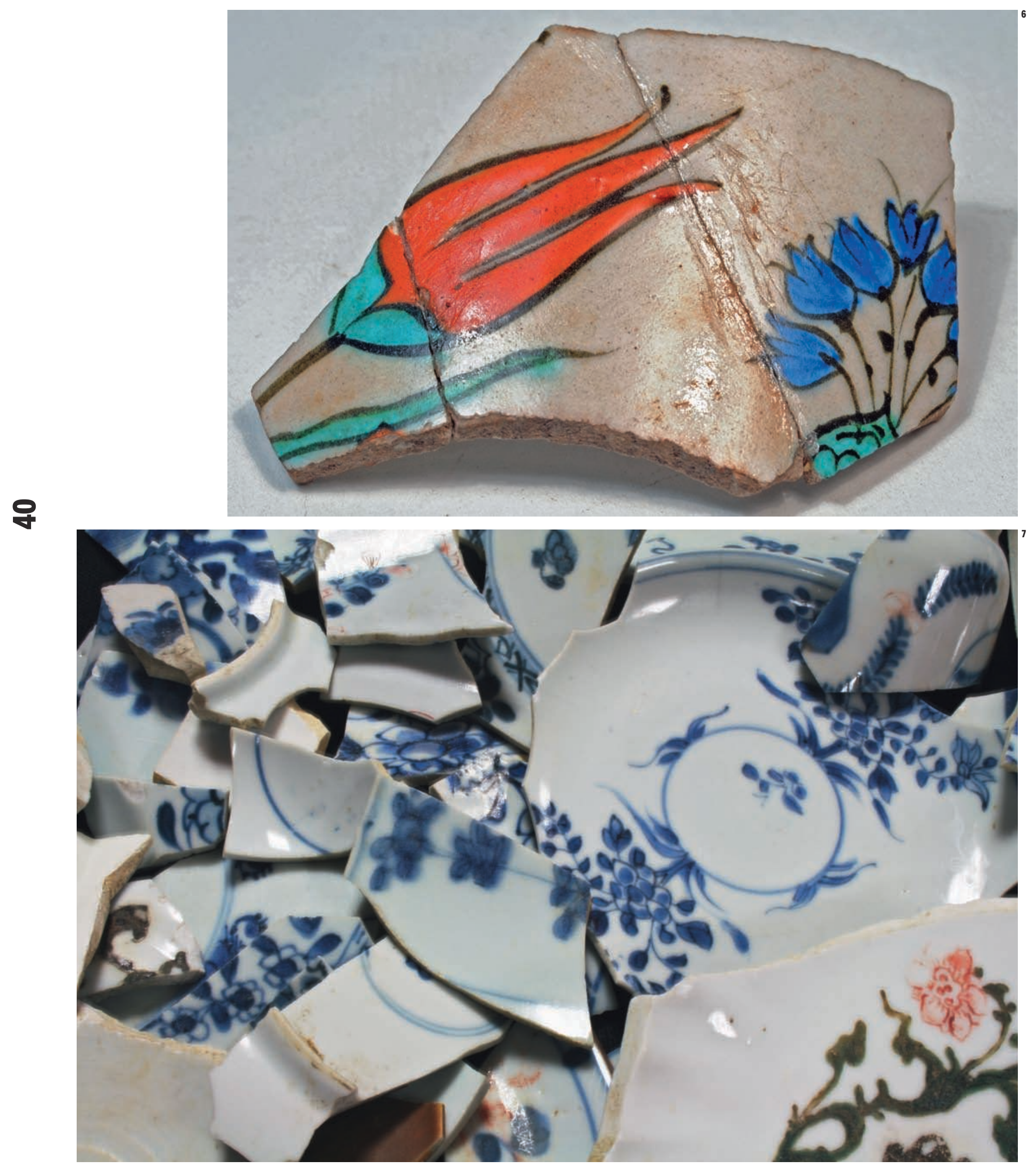


les céramiques sont un support idéal pour étudier l'évolution des goûts et les innovations. Mais, au stade actuel des études régionales, il est encore difficile de fixer avec assurance la part réservée alors à ces pièces. Approfondir le dépouillement des archives permettrait peut-être de prendre la mesure de l'arrivée des vaisselles et des produits de consommation exotiques dans la ville de Lyon, comme ce fut fait pour la Provence et le Languedoc.

D'ores et déjà, les quelques « chinoiseries » découvertes sur le site du parc Saint-Georges à Lyon sont révélatrices. Elles proviennent de dépotoirs issus d'un quartier que l'on peut alors considérer comme populaire, aux habitants peu fortunés. On notera dans les produits de terre cuite qui constituaient leur quotidien une volonté de répondre aussi à une mode considérée alors comme le fait de populations plus aisées. Les études céramologiques couplées à une analyse sociale permettront de nuancer les phases et les modes de démocratisation des phénomènes de consommation.

Références bibliographiques

AmOURIC H., Richez F., VAllauRi L., 1999: Vingt mille pots sous les mers. Le commerce de la céramique en Provence et Languedoc $d u X^{e}$ au XIX $X^{e}$ s., Catalogue d'exposition, Istres, 1999, Aix-en-Provence, Edisud. Amouric H., Horry A., VAYSSETtes J-L., 2009: «La naissance de la faïence moderne dans le Midi français ", in $\mathrm{VIII}^{e}$ Congreso Internacional de Ceramica Medieval en el Mediterraneo, Ciudad Real-Almagro, 27 février-3 mars 2006, Ciudad Real, p. 599-616.

Ayala G. (DIR.), 2013 : « Lyon, Saint-Georges. Archéologie, environnement et histoire d'un espace fluvial en bord de Saône », Documents d'archéologie française, $\mathrm{n}^{\circ} 106$.

HORRY A., 2001 : « La faïence à Lyon du XIV ${ }^{\mathrm{e}}$ au XVI ${ }^{\mathrm{e}}$ siècle l'apport des fouilles récentes », Archéologie du Midi médiéval, t. 19, p. 137-179.

Horry A., 2002 : « Découverte de faïences hispaniques lors des fouilles archéologiques récentes à Lyon ", in Le calife, le prince et le potier, Catalogue d'exposition, Lyon, musée des Beaux-Arts, 2 mars-22 mai 2002, Paris, Seuil, p. 228-229.

HORRY A., 2009: « Le vaisselier lyonnais du XIv e au $\mathrm{XVI}^{\mathrm{e}} \mathrm{s}$. : vers la naissance de la céramique moderne», in La cuisine et la table dans la France de la fin du Moyen Âge, Caen, Publications du CRAHM p. 299-316.

HORRY A., 2012a : « Entre Nord et Sud. Céramiques médiévales en Lyonnais et Dauphiné », in Atti del IX congresso internazionale sulla ceramica medievale nel Mediterraneo . Venezia, Scuola Grande dei Carmini 23-27 novembre 2009, Borgo san Lorenzo, All'Insegna del Giglio, p. 58-63.

Horry A., 2012b: Poteries de Lyon. 1500-1850. Morceaux choisis du quotidien à Saint-Georges, Lyon, Éditions lyonnaises d'art et d'histoire - Inrap.

THiriot J., 1991 : « Céramiques fines islamiques du midi de la France au bas Moyen Âge ", in Â cerâmica medieval do mediterraneo occidental, Actes du colloque de Lisbonne, 1987, Mértola, Campo arqueologico de Mértola, p. 285-303. 


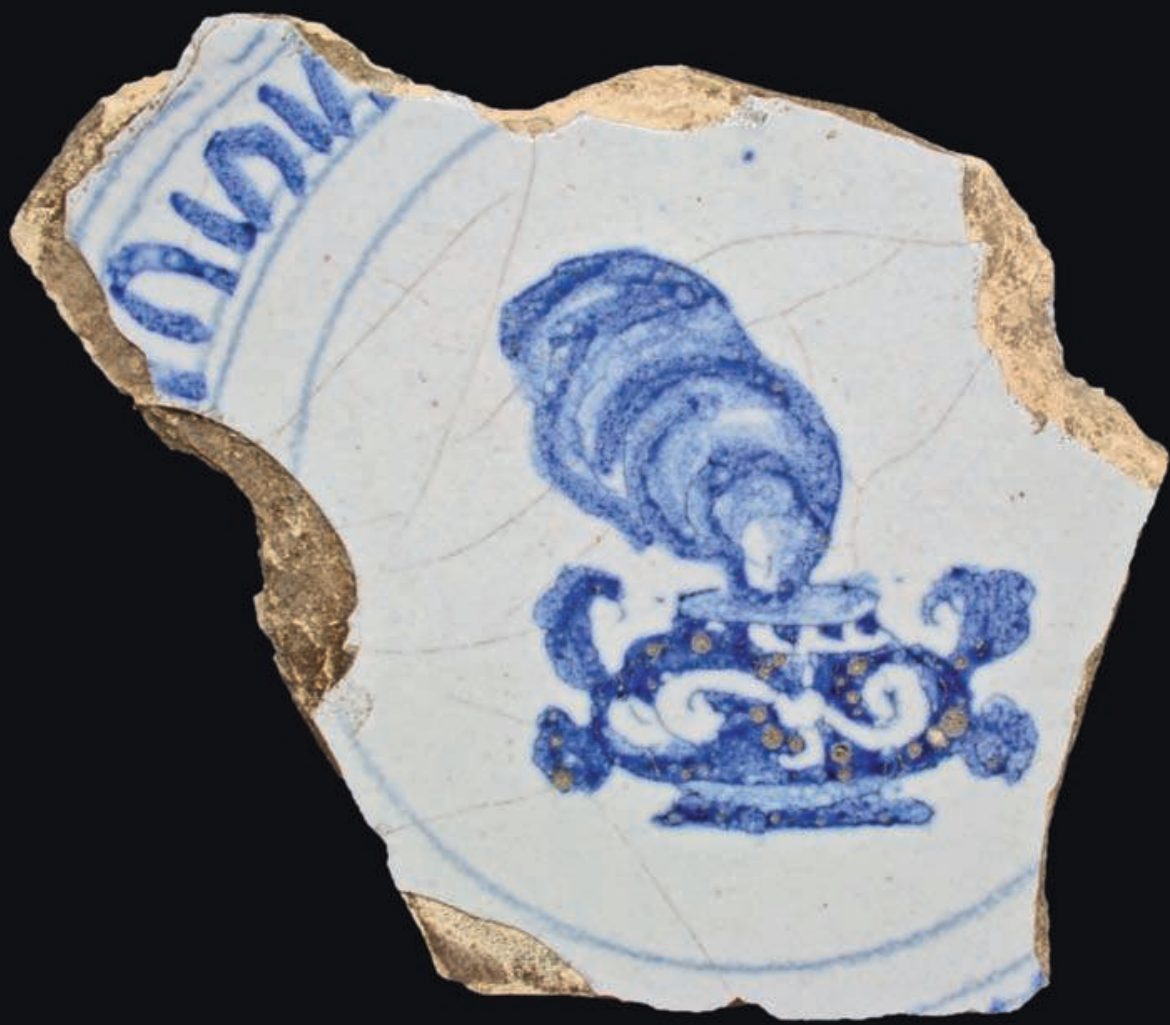

1. Une lampe d’Aladin sur un fond d'écuelle en fä̈ence lyonnaise du xvur siècle.

À $1 \mathrm{mode}$ de..

Si les fouilles archéologiques n'ont révélé qu'une présence rare de produits céramiques importés depuis des contrées lointaines comme l'Orient ou 1 'Trxtrême-Orient, en revanche l'étude des milliers d'objets découverts dans les dépotoirs de la période moderne à Iyon montre, par les styles ornementaux des « turqueries » et «chinoiseries », et parallèlement à d'autres régions de production en France, un goût certain pour un ailleurs réinventé. Ge fond d'écuelle en faience lyonnaise du xvirr siècle découverte sur le site du parc Saint-Georges ne porterait-il pas témoignage pour ce goût d'un Orient fabuleux par la représentation de la lampe d'Aladin. popularisée par l'orientaliste Antoine Galland au début du xvir' siècle via la traduction et l'édition des Mille et une nuits [ill. 1].

Turqueries

Le goût et l'intérêt pour 1'Orient sont connus dès le début de la période moderne. Ies prestigieuses céramiques d'Iznik, emblématiques du xvr siècle, arrivent jusqu'en région lyonnaise, produits de grand luxe originaires des contrées ottomanes. Au sein de fleurs variées qui servent de décors à ces divers supports en terre cuite (plats, chopes, bouteilles...), les tulipes occupent une place de choix. Ces fleurs qui vont être exportées en Furope dès le xvrr siècle suscitent un engouement sans précédent et une véritable passion qui atteindra très rapidement les arts décoratifs. Hlles symbolisent alors largement les contrées exotiques et font aussi partie des produits de luxe. Flles vont progressivement « fleurir » sur les céramiques et en particulier sur les faiences. On pense bien sûr à la culture de la fleur aux Pays-Bas et aux prestigieuses manufactures de Delft qui vont largement en utiliser le motif. Ia passion pour ces fleurs est également à l'origine de la création par les faïenciers de récipients destinés à les présenter sous la forme de bouquets ou à l'unité.

I'étude des céramiques des xvIr et xvIIr ${ }^{\mathrm{e}}$ siècles découvertes à Iyon, dans les fouilles archéologiques, permet d'évoquer, dans une certaine adaptation, ce phénomène d'engouement pour la tulipe. Ia découverte de milliers de tessons de céramique glaçurée décorée à l'aide d'engobes illustre 1'adaptation de motifs floraux, connus alors en majorité sur les faiences, à des poteries plus populaires et diffusées plus largement. On ne compte plus les centaines d'écuelles, de plats, d'assiettes et de cruches qui présentent des bouquets de tulipes [ill. 2 et 3] aux côtés d'autres fleurs locales. Des fleurs imaginaires qui copient ou s'inspirent des tulipes font également partie du registre décoratif inventé et appliqué par les potiers régionaux. Cette ornementation des céramiques engobées, que l'on appelle aussi les terres vernissées, constitue un avatar de la mode pour l'exotisme, en constante évolution, qui se diffuse parmi la population de Iyon. Ies terres vernissées lyonnaises des $\mathrm{xvII}^{\mathrm{e}}$ et $\mathrm{xvII}^{\mathrm{e}}$ siècles offrent parfois des objets qui ne sont pas loin de rappeler les vases à fleurs ou tulipiers qui étaient produits par de nombreuses manufactures de faiences. Il s'agit de pique-fleurs au profil original, sortes de petits vases percés pour $y$ mettre des bouquets. Ces derniers peuvent être finalement les preuves d'une probable « démocratisation » d'objets du quotidien réservés jusqu'alors à une certaine élite sociale. 


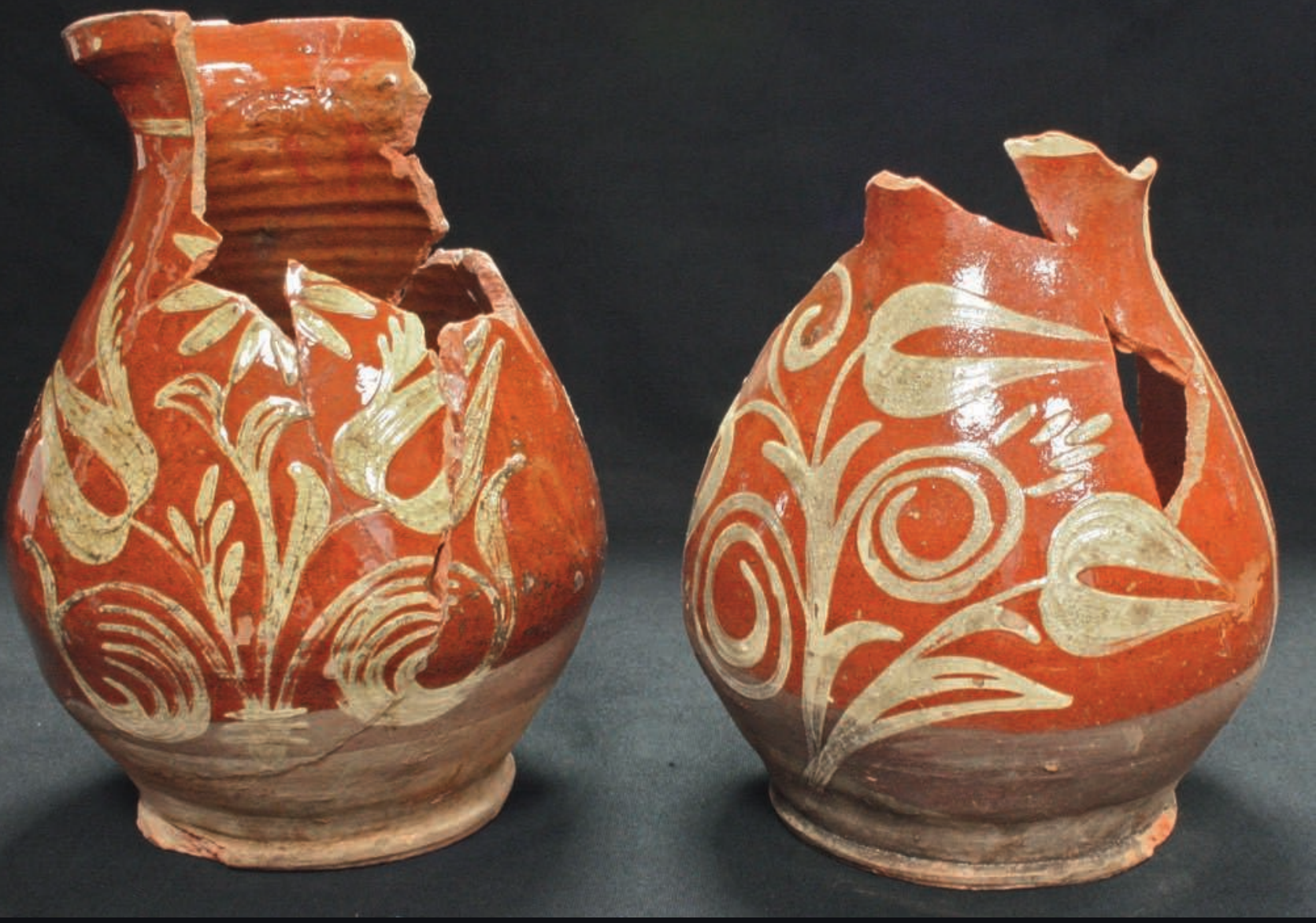

9P

2.Cruches en céramique glaçurée décorées aux engobes.

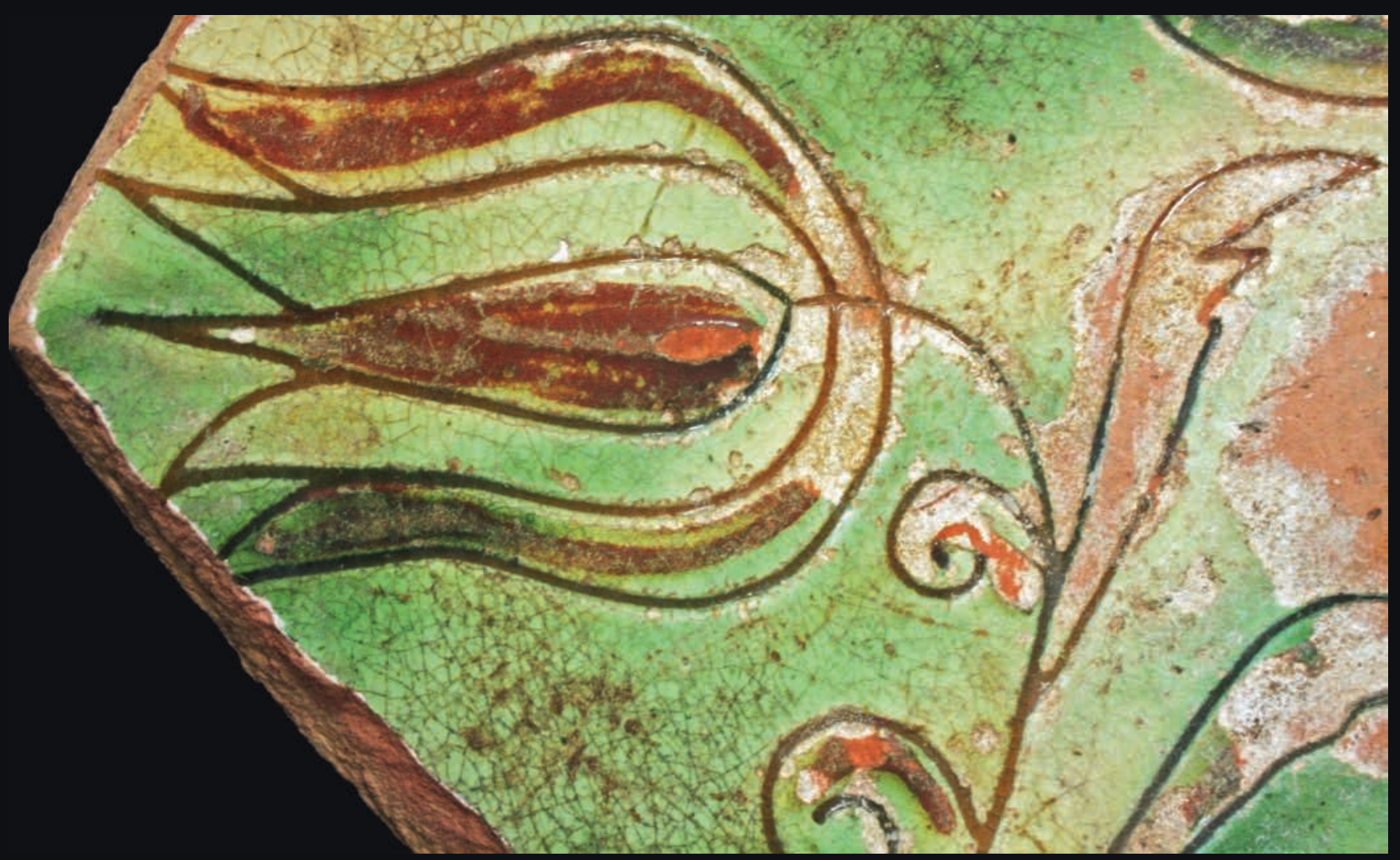

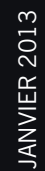




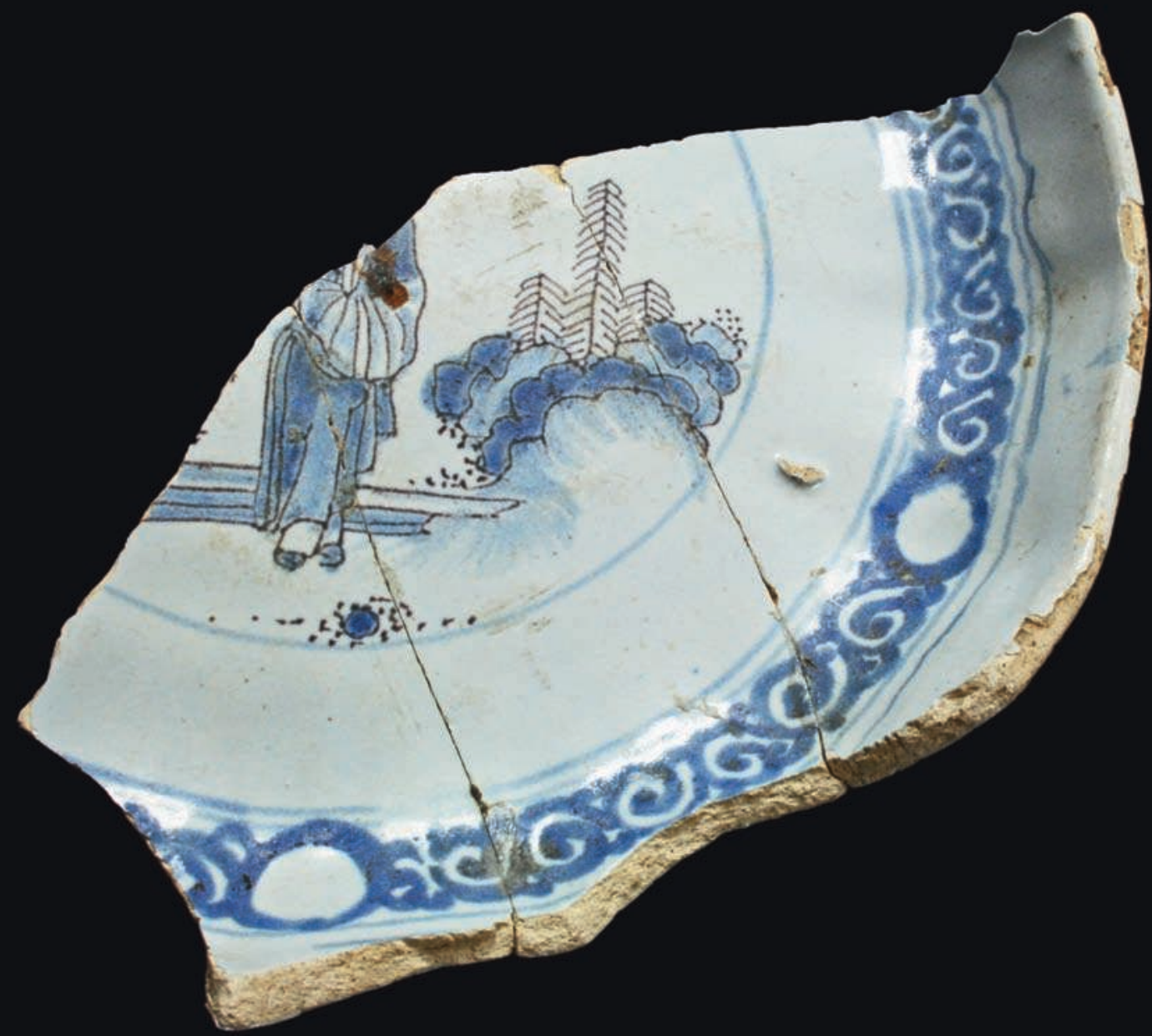

4. Une coupe en faïence de Nevers aux motifs chinois.

Chinoiseries

Les découvertes des dépotoirs lyonnais de la seconde moitié du xvIr ${ }^{e}$ siècle et surtout du xvII ${ }^{e}$ siècle livrent pléthore de faiences qui permettent aussi d'aborder une des autres formes de l'exotisme. I'utilisation du langage figuré d'une Chine imaginaire et qui fascine est désormais fréquente sur des vaisselles de table ou autres objets du quotidien. De nombreuses manufactures de faïences produisent dès lors des vaisselles dans le goût de la Ghine soit par le biais de copies soit par la création de motifs originaux. Ies fouilles du parc Saint-Georges livrent une collection conséquente de faiences qui illustrent l'attrait pour ce répertoire. Ces céramiques proviennent de dépotoirs d'un quartier populaire situé au bord de la Saône. Divers objets contribuent à donner un peu de luxe à la table, à la fois pour la décorer et pour présenter et servir des mets particuliers (boissons chaudes, confiseries, pâtisseries...). De nombreuses coupes ou objets divers en faience de Nevers de la fin du xvir ${ }^{e}$ siècle et du xviri siècle [ill. 4] sont ornés de motifs qui rappellent les décors bleus et blancs de Chine (Horry, 2012b, p. 104-105) et qui mettent en scène des personnages dans des paysages simplifiés et imaginaires. D'autres vaisselles plus prestigieuses et originaires de Delft aux Pays-Bas comme cette assiette ornée de cceurs et de fleurs [ill. 5] ou cette coupe au décor cachemire [ill. 6] ont pu constituer les pièces exceptionnelles du décor de quelque maison modeste de ce quartier. On peut observer également sur de menus fragments des personnages aux costumes chinois [ill. 7] qui sont autant de témoignages de cet engouement pour des images exotiques comme aussi sur cette assiette en faience de Iyyon au décor polychrome [ill. 8].

Si les " chinoiseries » se taillent donc la part belle au cceur des vaisselles exotiques du xvmr siècle, on peut également leur joindre d'autres productions dont les décors ont dû largement contribuer à développer l'imaginaire et le fantastique par le biais d'un exotisme ornemental. On pense bien sûr aux faiences de Moustiers avec les fameux décors de grotesques [ill. 9] qui ont été créés par le faiencier Joseph Olérys. Ces motifs trouvent leur origine dans les décors chinois présents sur les faiences d'Alcora en Fspagne. Ces personnages fantastiques, qui participent d'une mode affectant arts décoratifs, peinture, opéra..., ornent aussi bon nombre de faiences de production lyonnaise vers le premier quart du xviri siècle [ill. 10]. 


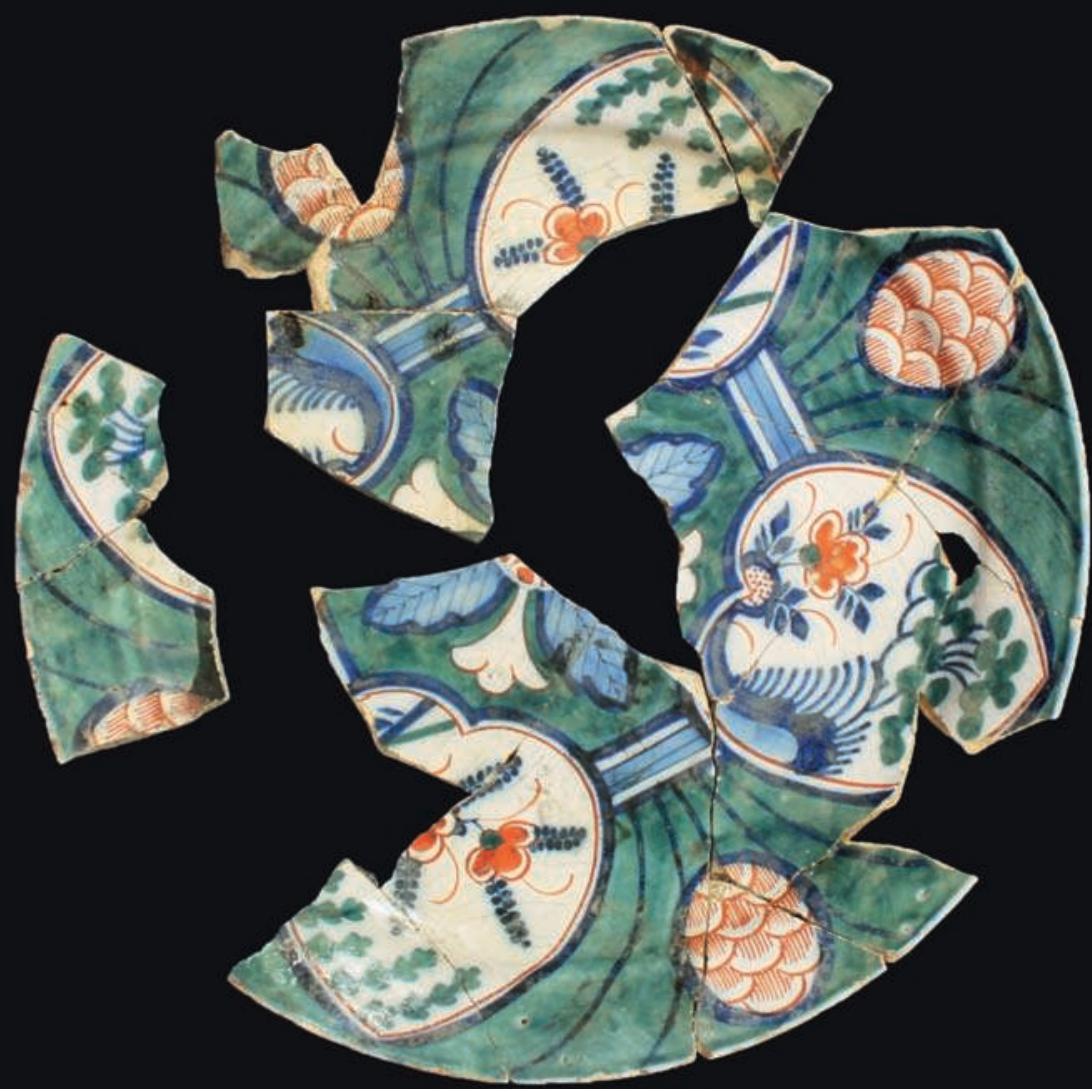

5. Assiette en faïence de Delift décorée « aux quatre ccesurs ॥.

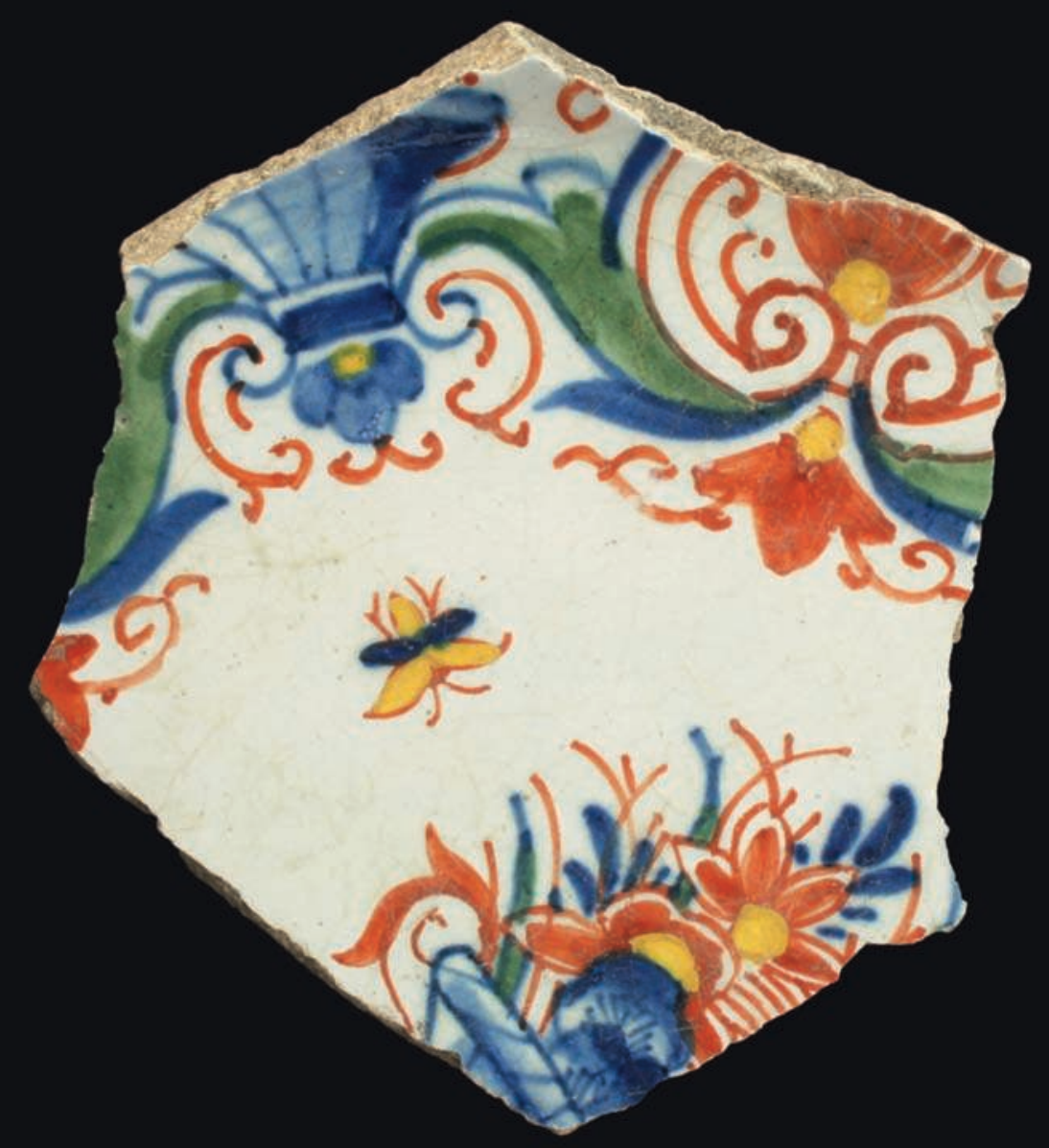

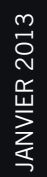

6. Fragment d'une coupe de Delft au décor dift " c cachemire ॥. 


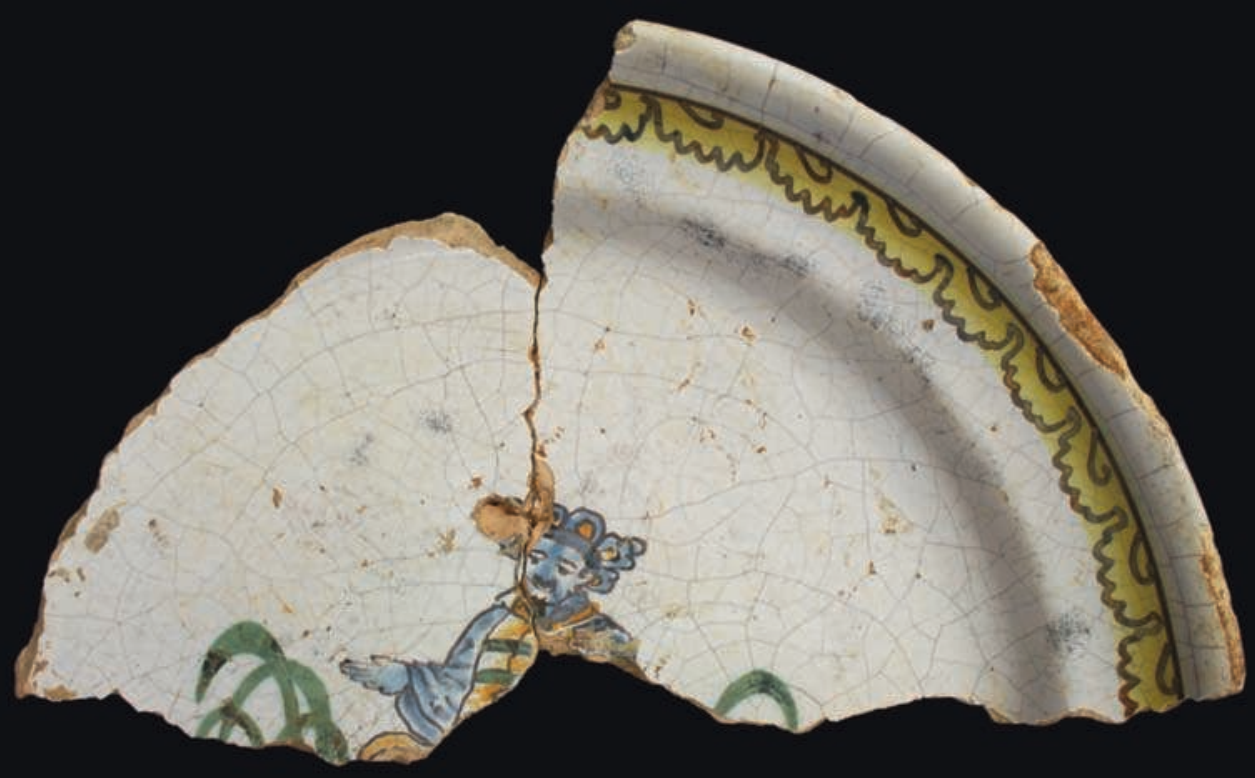

7. Fragment d'assiette représentant un personnage en costume chinois.

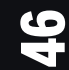

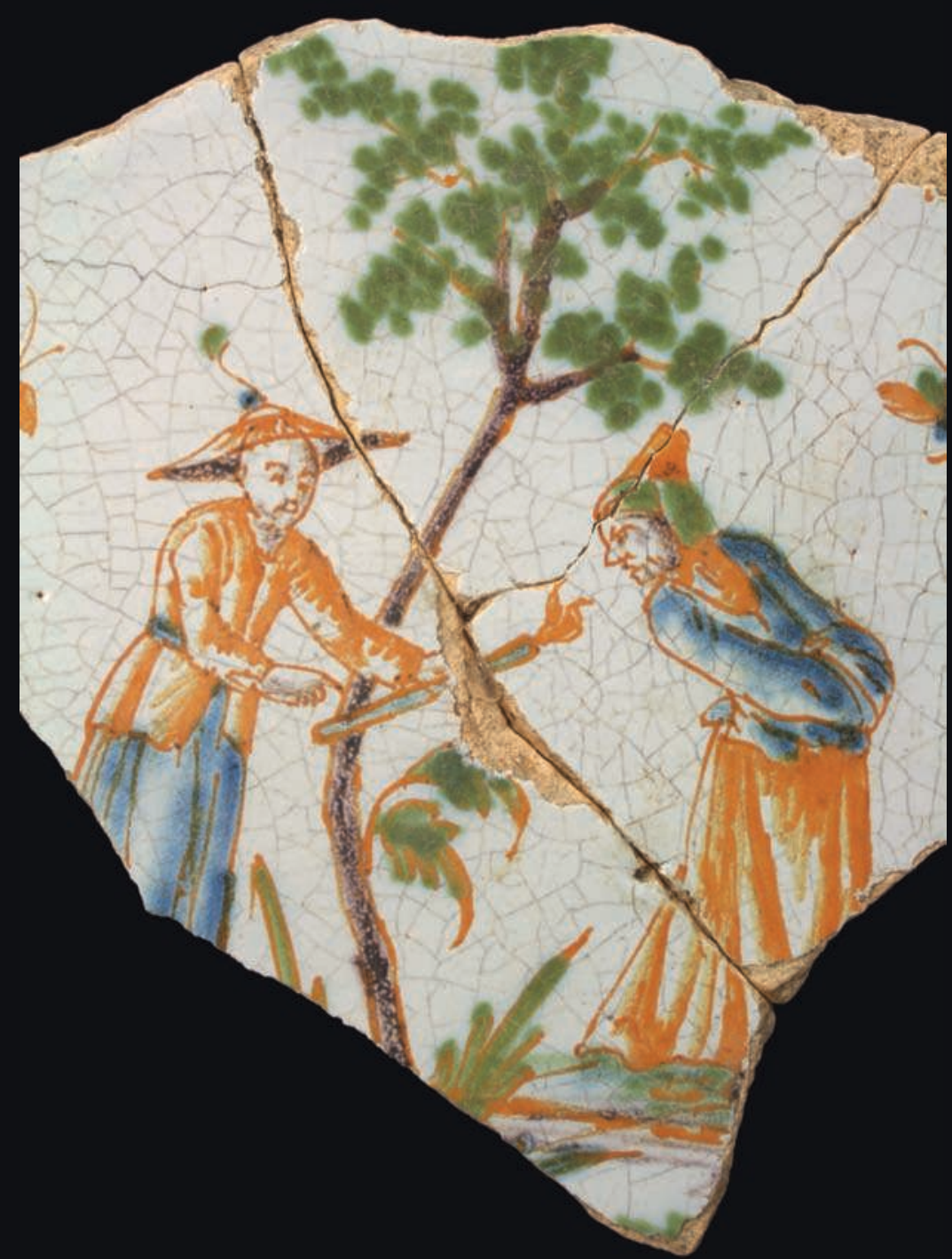

8.Assiette en faïence de Lyon au décor polychrome. 


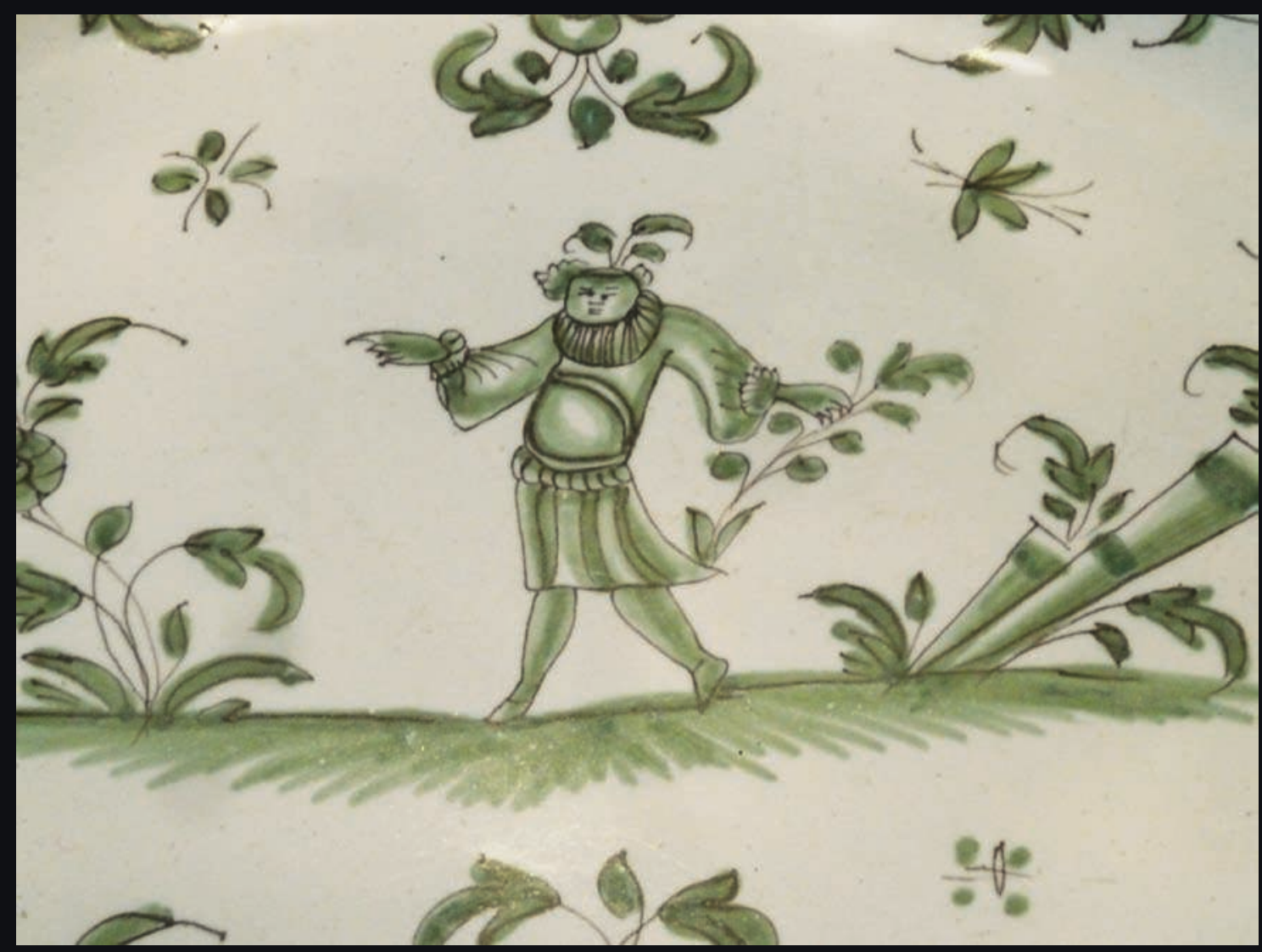

9. Un Chinois sur une faïence de Moustiers au décor de grotesques.

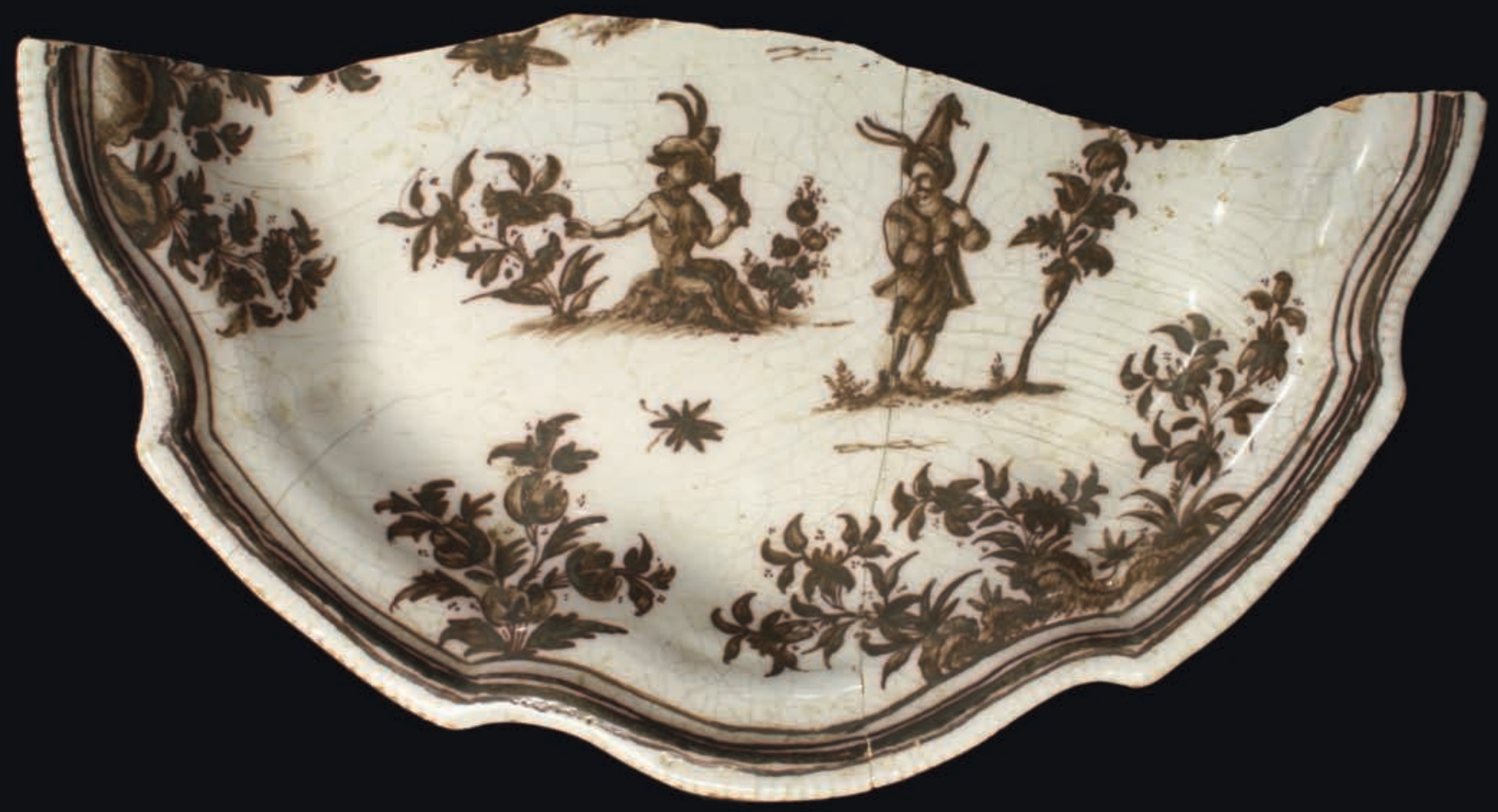

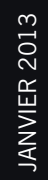

